\title{
Associações entre ingestão energética, proteica e de fósforo em pacientes portadores de doença renal crônica em tratamento hemodialítico
}

\author{
Associations between energy, protein, and phosphorus intakes in \\ patients with chronic kidney disease on hemodialysis
}

\begin{abstract}
Autores
Denise Entrudo Pinto ${ }^{1}$

Laura Sulzbach

Ullmann ${ }^{1}$

Mariana Marroni

Burmeister $^{2}$

Ivan Carlos Ferreira

Antonello ${ }^{3}$

Alessandra Pizzato ${ }^{1}$

'Pontifícia Universidade Católica do Rio Grande do Sul, Faculdade de Enfermagem, Nutrição e Fisioterapia - Porto Alegre, RS, Brasil ${ }^{2}$ Associação Hospitalar Moinhos de Vento Porto Alegre, RS, Brasil ${ }^{3}$ Hospital São Lucas da Pontifícia Universidade Católica do Rio Grande do Sul - Porto Alegre,

RS, Brasil
\end{abstract}

Data de submissão:18/03/2009 Data de aprovação: 01/10/2009

Correspondência para: Denise Entrudo Pinto Rua Veríssimo Rosa, 390 Bairro Partenon- Porto Alegre/RS

CEP: 90610-280.

E-mail: denidep@yahoo. com.br

Declaramos a inexistência de conflitos de interesse.

\section{Resumo}

Introdução: A nutrição desempenha papel fundamental nas doenças renais. A recomendação nutricional deve ofertar uma dieta hiperproteica, adequada em energia e fósforo segundo o Kidney Disease Outcomes Quality Initiative (K/DOQI). É necessário controlar e/ou prevenir as complicações da Doença Renal Crônica (DRC), pois ela impõe desafios clínicos diretamente ligados ao estado nutricional. Objetivo: Investigar as associações entre a ingestão energética, proteica e de fósforo em pacientes em hemodiálise (HD). Pacientes e Métodos: Estudo observacional envolvendo 72 pacientes em HD, em dois hospitais de Porto Alegre/RS/Brasil. Foram coletados dados referentes ao perfil antropométrico (peso, altura e índice de massa corporal-IMC) e do registro alimentar de três dias (ingestão de energia, proteína e fósforo). $\mathrm{O}$ teste de correlação de Spearman foi utilizado para avaliar as associações entre as variáveis do registro alimentar $(\mathrm{p}<0,05)$. Resultados: A ingestão energética, proteica e de fósforo foi, respectivamente, de: $28 \pm 10,0 \mathrm{kcal} / \mathrm{kg}$, $1,1 \pm 0,4 \mathrm{~g} \mathrm{ptn} / \mathrm{kg}, 958 \pm 374 \mathrm{mg} / \mathrm{dia}$, estando os níveis de energia e proteína abaixo das recomendações do K/DOQI. Observou-se correlação positiva forte entre as variáveis. Discussão: O perfil no tratamento nutricional é fundamental, pois houve correlações entre os nutrientes. É importante salientar que as recomendações do K/DOQI não conseguem ser atingidas pelos pacientes. Conclusão: Há associação positiva entre a ingestão de energia, proteína e fósforo na alimentação de pacientes em HD. O controle desses nutrientes na dieta é essencial, pois auxilia no controle das complicações relacionadas com a DRC. Assim, a avaliação nutricional é importante nas unidades de diálise para definir o perfil dietético dos pacientes e que seja feito o ajuste de suas prescrições.

Palavras-chave: insuficiência renal crônica, hemodiálise, energia, proteína e fósforo.

[J Bras Nefrol 2009;31(4):269-276]@Elsevier Editora Ltda.

\section{Abstract}

Introduction: Nutrition plays an important role in kidney disease. The nutritional recommendation is to offer a high-protein diet, adequate in energy and phosphorus according to the Kidney Disease Outcomes Quality Initiative (K/DOQI) guidelines. Control and/or prevention of the complications of chronic kidney disease (CKD) are essential, because CKD poses clinical challenges directly related to the nutritional status. Objective: To investigate the possible associations between energy, protein, and phosphorus intakes in hemodialysis (HD) patients. Methods: Observational study involving $72 \mathrm{HD}$ patients from two hospitals in the city of Porto Alegre, Brazil. Anthropometric data [weight, height, body mass index (IMB)] were collected and three-day food intake (daily energy, protein, and phosphorus intakes) was recorded. Spearman correlation was used to evaluate associations between food intake variables $(\mathrm{p}<0.05)$. Results: The energy, protein, and phosphorus intakes were $28 \pm$ $10 \mathrm{kcal} / \mathrm{kg} / \mathrm{day}, 1,1 \pm 0.4 \mathrm{~g}$ protein $/ \mathrm{kg}$, and $958 \pm 374 \mathrm{mg} /$ day, respectively, and the first two were below the K/DOQUI recommendations. A strong positive correlation was observed between the variables. Discussion: Assessing the nutritional profile is essential, because correlations between nutrients occur. It is worth emphasizing that the K/DOQI guidelines are not met by the patients. Conclusion: A positive association between the intakes of energy, protein, and phosphorus is observed in the diet of HD patients. The dietary control of these nutrients is essential to manage CKD complications. Therefore, nutritional evaluation in dialysis units is crucial to define the dietary profile of patients and to adjust their prescriptions.

Keywords: chronic renal failure, hemodialysis, energy, protein, phosphorus. 


\section{INTRODUÇÃO}

A nutrição desempenha um importante papel na avaliação e no tratamento das doenças renais. $\mathrm{O}$ aconselhamento dietético individualizado deve estar associado a programas de educação nutricional, visando a auxiliar no controle e na prevenção das complicações da Doença Renal Crônica (DRC), uma vez que ela, em suas várias etapas, impõe desafios clínicos diretamente ligados ao estado nutricional. ${ }^{1}$

$\mathrm{Na}$ avaliação nutricional, é essencial certificar-se da eficiência do procedimento dialítico, medida por meio do cálculo do Kt/V de ureia. Inadequações nesse procedimento podem resultar em um estado urêmico, tendo como consequências manifestações que podem interferir no perfil nutricional do paciente, como náuseas, vômitos e anorexia. ${ }^{2}$

Durante o procedimento hemodialítico, ocorrem perdas significativas de aminoácidos para o dialisato, sendo fundamental um aporte proteico adequado para suprir estas demandas e evitar um quadro de desnutrição energética proteica (DEP). ${ }^{3}$ A DEP ocorre quando há oferta inadequada de proteínas ou calorias, e seu impacto é desfavorável no prognóstico da DRC. Segundo o National Cooperative Dialysis Study, os pacientes com uma ingestão menor do que $0,8 \mathrm{~g} \mathrm{ptn} / \mathrm{kg} / \mathrm{dia}$, estimada pelo aparecimento de nitrogênio urinário (Protein Nitrogen Appearance - PNA), apresentam aumento na taxa de morbidade. ${ }^{4}$ Dessa forma, as recomendações proteicas e energéticas estão aumentadas, a fim de promover um balanço nitrogenado neutro ou positivo, sendo de, aproximadamente, $1,2 \mathrm{~g}$ de proteína $/ \mathrm{kg} / \mathrm{dia} \mathrm{e}$ $30-35 \mathrm{kcal} / \mathrm{kg} /$ dia para manutenção do peso. ${ }^{5}$

Por outro lado, essa maior necessidade proteica, que tem como objetivo prevenir a desnutrição, dificulta o tratamento do quadro de hiperfosfatemia, comumente presente na DRC devido à deficiência de calcitriol e ao descontrole do balanço cálcio-fósforo, com consequente aparecimento de hiperparatireoidismo secundário, que pode determinar o desenvolvimento de doença óssea, influenciando na velocidade de progressão da DRC. ${ }^{6,78}$ Os níveis séricos elevados de fósforo são resultantes de três fatores principais: a ingestão excessiva de fósforo, a redução da depuração de fósforo e o estado da remodelação óssea, e estão associados a maiores taxas de morbidade e mortalidade relacionados com eventos cardiovasculares. ${ }^{5}$ Assim, frequentemente é necessária a utilização de quelantes - compostos que se ligam ao fósforo do alimento no intestino, reduzindo sua absorção. A quantidade de quelantes prescrita depende da quantidade de fósforo na alimentação., ${ }^{910,11}$
A partir das questões apontadas, delineamos o presente estudo com o objetivo de investigar as possíveis associações entre a ingestão de energia, proteína e fósforo em pacientes renais crônicos em tratamento dialítico, e caracterizar o perfil antropométrico dos pacientes.

\section{Pacientes e Métodos}

Foi realizado um estudo observacional com base em dados secundários à dissertação de mestrado intitulada: "Ingestão e níveis séricos de potássio, fósforo e cálcio de pacientes com insuficiência renal crônica em tratamento hemodialítico" ${ }^{13} \mathrm{O}$ estudo foi realizado nos hospitais São Lucas, da Pontifícia Universidade Católica do Rio Grande do Sul (HSL), e Moinhos de Vento (HMV), de Porto Alegre/RS.

Ambas as instituições seguem as orientações nutricionais preconizadas pelo K/DOQI. Entretanto, existem diferenças na periodicidade do acompanhamento com os nutricionistas: no HMV, a visita é semanal e, no HSL, é diária.

Foram analisados todos os pacientes com DRC em tratamento hemodialítico que fizeram parte do estudo anteriormente citado: 26 no HSL e 46 no HMV. Os critérios de inclusão foram: pacientes de ambos os sexos; maiores de 18 anos de idade; não hospitalizados; em tratamento hemodialítico há pelo menos três meses; passíveis de avaliação antropométrica por meio de métodos tradicionais de verificação de peso e altura (para cálculo do índice de massa corporal - IMC); sem infecção; sem uso de antimicrobianos; que devolveram o registro alimentar preenchido corretamente. Os pacientes usavam quelantes de fósforo conforme a necessidade, a prescrição de seus médicos e seu próprio nível de aderência, não havendo interferência dos pesquisadores que alterasse sua rotina.

Foram coletados os dados referentes ao perfil nutricional: antropométrico e dietético. Com relação aos dados antropométricos, foram avaliados: peso, altura e IMC. Foi calculado o peso ideal para os pacientes que foram classificados fora dos parâmetros de normalidade pelo IMC, por meio da seguinte fórmula: $18,5 \mathrm{~kg} / \mathrm{m}^{2} \mathrm{x}$ altura $(\mathrm{m})^{2}$, para aqueles abaixo da normalidade (sendo utilizado o valor do IMC mínimo para eutrofia de $18,5 \mathrm{~kg} /$ $\left.\mathrm{m}^{2}\right) ;$ e $24,9 \mathrm{~kg} / \mathrm{m}^{2} \mathrm{x}$ altura $(\mathrm{m})^{2}$ para aqueles acima do peso (utilizado-se o valor do IMC máximo para eutrofia de $24,9 \mathrm{~kg} / \mathrm{m}^{2}$ ). Este peso ajustado foi utilizado no cálculo da ingestão calórica e proteica por $\mathrm{kg} / \mathrm{dia}^{2}$. 
Para avaliar o perfil dietético, foi utilizado o registro alimentar de três dias, sendo dias alternados: um dia da semana sem o tratamento hemodialítico, um dia da semana com o tratamento e um domingo, por ser atípico, no período de fevereiro a abril de 2007. Foram coletados dados referentes à ingestão de calorias (totais e kg/dia), proteínas (totais, porcentagem de proteína do Valor Calórico Total (VCT), gramas por $\mathrm{kg} / \mathrm{dia}$ e \% de Alto Valor Biológico - AVB) e fósforo. O registro alimentar seguiu cuidadosamente os critérios para maior confiabilidade dos dados: instruções para o preenchimento do registro alimentar; orientação das porções e especificação do tipo de alimentos; e horários para anotação do consumo. Os participantes foram questionados quanto ao apetite, sendo classificados em três categorias: apetite diminuído, normal e aumentado.

Os registros alimentares foram analisados separadamente pelo programa de apoio à nutrição Nutwin -, do Centro de Informática em Saúde da Escola Paulista de Medicina, versão 1,5/2002. O consumo de calorias, proteínas e fósforo foi comparado às recomendações do K/DOQI, que estabelecem cotas de ingestão diária dos vários nutrientes para os pacientes em tratamento hemodialítico: $35 \mathrm{kcal} / \mathrm{kg}$, $1,2 \mathrm{~g}$ ptn $/ \mathrm{kg}$, mínimo de $50 \%$ de proteína de AVB, e $800-1000 \mathrm{mg}$ de fósforo. ${ }^{12}$

Com relação aos parâmetros bioquímicos, foram avaliados os níveis de fósforo sérico (valores de referência de 3,5 a $5,5 \mathrm{mg} / \mathrm{dL}$ ) para considerar possíveis riscos de hiperfosfatemia ${ }^{12}$ e adequação da diálise. Foi utilizado o cálculo do Kt/V de ureia, sendo considerados adequados valores iguais ou superiores a 1,2. ${ }^{14,15}$

Foi realizada a análise descritiva das características gerais da população do estudo (sexo, idade, peso, altura, IMC), dos parâmetros dietéticos (ingestão de energia, proteínas e fósforo), bioquímicos (fósforo séricos e $\mathrm{Kt} / \mathrm{V}$ ) e a utilização de quelantes de fósforo. Foi realizado o teste de correlação de Spearman entre as variáveis: calorias totais, calorias por $\mathrm{kg} / \mathrm{dia}$, proteínas totais, proteínas em gramas por $\mathrm{kg} / \mathrm{dia}$ e fósforo. A significância estatística foi definida como $\mathrm{p}<0,05$. Os dados foram tabulados e analisados com auxílio do programa Statistic Package for Social Sciences (SPSS), versão 13.0 , e apresentados na forma de média \pm desvio-padrão $(\mathrm{M} \pm \mathrm{DP})$ e porcentagem $(\%)$.

Foram respeitados os aspectos éticos envolvidos na pesquisa com seres humanos, considerando-se as questões expressas na resolução 196/96 do CNSMS. ${ }^{16} \mathrm{O}$ estudo foi aprovado pelo comitê de ética do Hospital São Lucas e do Instituto de Educação em Pesquisa do Hospital Moinhos de Vento.

\section{Resultados}

No presente estudo, foram avaliados 72 pacientes com DRC em tratamento hemodialítico, sendo dois excluídos por subestimação dos registros alimentares. Destes, 46 pacientes eram do HMV e 26 eram do HSL. Havia 47 homens e 25 mulheres, com média de idade de $61 \pm 15$ anos.

Com relação ao perfil antropométrico, a média de peso $(\mathrm{kg})$, altura $(\mathrm{m})$ e IMC $\left(\mathrm{kg} / \mathrm{m}^{2}\right)$ foi de: $68,77 \pm 14$ $\mathrm{kg}, 1,65 \pm 0,8 \mathrm{~m}$ e $25,2 \pm 5 \mathrm{~kg} / \mathrm{m}^{2}$, respectivamente. Não houve diferenças significantes dos pacientes entre as instituições, sendo que, em ambos hospitais, observou-se média de IMC acima do recomendado $\left(>24,9 \mathrm{~kg} / \mathrm{m}^{2}\right): 25,1 \pm 4 \mathrm{~kg} / \mathrm{m}^{2}$ no HMV e $25,4 \pm 5 \mathrm{~kg} / \mathrm{m}^{2}$ no HSL

Observou-se também que 13,8\% referiram diminuição no apetite, $63,8 \%$ apetite normal e 17,5\% apetite aumentado. Em relação à utilização de quelantes de fósforo, $79,2 \%$ dos pacientes não os utilizavam.

Os registros alimentares de três dias dos pacientes de ambos os hospitais foram comparados com as recomendações do K/DOQI. ${ }^{12}$ Observou-se que, em geral, a ingestão energética e proteica diária estava abaixo do recomendado, em torno de $28 \pm 10 \mathrm{kcal} / \mathrm{kg} / \mathrm{dia}$ e $1,1 \pm 0,4 \mathrm{~g} \mathrm{ptn} / \mathrm{kg}$, respectivamente, e o fósforo esteve dentro da normalidade $(958 \pm 374 \mathrm{mg} / \mathrm{dia})$.

Separando os participantes por metas alcançadas na dieta quanto à ingestão inadequada de nutrientes, observou-se que $61,6 \%$ dos participantes tiveram uma ingestão proteica abaixo do recomendado. Com relação às calorias e ao fósforo, essa ingestão foi de $79,1 \%$ e $41,6 \%$, respectivamente. Entretanto, $20,8 \%, 37,5 \%$ e $38,8 \%$, respectivamente, ingeriram acima do preconizado com relação a calorias, proteínas e fósforo.

Os resultados dos coeficientes de correlação de Spearman entre as variáveis do registro alimentar de três dias relacionados com a ingestão energética, proteica e de fósforo da dieta de pacientes em HD estão demonstrados na Tabela 1. Observou-se correlação muito forte entre a ingestão de energia ( $\mathrm{kcal} \mathrm{e} \mathrm{kcal} / \mathrm{kg}$ ), proteína (g e g/kg) e fósforo (mg) (Gráficos 1 e 2). Entretanto, foi encontrada uma correlação negativa baixa $\left(r_{s}=-0,294, p<0,05\right)$ entre a energia e a porcentagem de proteína do VCT.

$\mathrm{Na}$ Tabela 2, estão descritos os resultados dos coeficientes de correlação entre as variáveis relacionadas à ingestão de proteína de $\mathrm{AVB}$ e a ingestão energética, proteica e de fósforo do registro alimentar de três dias. 


\begin{tabular}{|ccc|}
\hline Tabela 1 & $\begin{array}{l}\text { COEFICIENTE DE CORRELAÇĀO DE SPEARMAN ENTRE VARIÁVEIS RELACIONADAS COM INGESTÃO ENERGÉTICA, } \\
\text { PROTEICA E DE FÓSFORO DO REGISTRO ALIMENTAR DE TRÊS DIAS }\end{array}$ & Energia (Kcal/kg) \\
\hline & & \\
\hline Proteína & $0,784^{\mathrm{a}}$ & $0,684^{\mathrm{a}}$ \\
\hline $\mathrm{g}$ & $0,720^{\mathrm{a}}$ & $0,812^{\mathrm{a}}$ \\
\hline g/kg & $-0,299^{\mathrm{b}}$ & $-0,294^{\mathrm{b}}$ \\
\hline FCT & $0,824^{\mathrm{a}}$ & $0,703^{\mathrm{a}}$ \\
\hline
\end{tabular}

Nota: amostra de 72 pacientes. ${ }^{a} \mathrm{p}<0,01 ;{ }^{b} p<0,05$. Onde: $\mathrm{kcal}=$ calorias; $\mathrm{kcal} / \mathrm{kg}=$ calorias/quilo; $\mathrm{g}=\mathrm{grama} ; \mathrm{g} / \mathrm{kg}=\mathrm{gramas} / \mathrm{quilo}$; \%VCT: porcentagem de proteína do valor calórico total diário; $\mathrm{mg} / \mathrm{dia}=$ miligramas/dia. Resultados apresentados na forma de $r_{\mathrm{s}}$. Teste de correlação de Spearman.

\begin{tabular}{|c|c|}
\hline \multirow[t]{2}{*}{ Tabela 2} & $\begin{array}{l}\text { COEFICIENTE DE CORRELACCÃO DE SPEARMAN ENTRE VARIÁVEIS RELACIONADAS À INGESTÃO DE PROTEÍNA DE ALTO } \\
\text { VALOR BIOLÓGICO COM INGESTÃO ENERGÉTICA, PROTEICA E DE FÓSFORO DO REGISTRO ALIMENTAR DE TRÊS DIAS }\end{array}$ \\
\hline & $A \vee B(g)$ \\
\hline \multicolumn{2}{|l|}{ Energia } \\
\hline Kcal & $-0,297^{b}$ \\
\hline $\mathrm{Kcal} / \mathrm{kg}$ & $-0,237^{b}$ \\
\hline \multicolumn{2}{|l|}{ Proteína } \\
\hline g & $0,144^{b}$ \\
\hline $\mathrm{g} / \mathrm{kg}$ & $0,169 b$ \\
\hline \multirow{2}{*}{$\%$ VCT } & $0,638^{a}$ \\
\hline & \\
\hline $\mathrm{mg} / \mathrm{dia}$ & $0,22^{b}$ \\
\hline
\end{tabular}

Nota: amostra de 72 pacientes. ${ }^{a} \mathrm{p}<0,01 ;{ }^{\mathrm{b}} \mathrm{p}<0,05$. Onde: $\mathrm{kcal}=$ calorias; $\mathrm{kcal} / \mathrm{kg}=$ calorias/quilo; $\mathrm{g}=$ grama; $\mathrm{g} / \mathrm{kg}=$ gramas/quilo; \%VCT: porcentagem de proteína do valor calórico total diário; $\mathrm{mg} / \mathrm{dia}=$ miligramas/dia. Resultados apresentados na forma de $\mathrm{r}_{\mathrm{s}}$. Teste de correlação de Spearman.

Gráfico 1. Coeficiente de correlação de Spearman entre a ingestão diária de fósforo $(\mathrm{mg})$ e energia $(\mathrm{kcal} / \mathrm{kg})$.

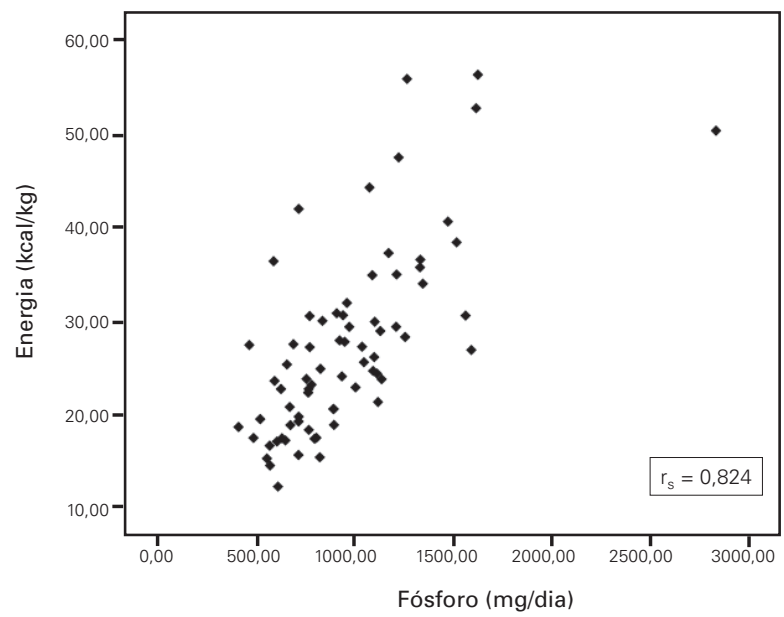

Gráfico 2. Coeficiente de correlação de Spearman entre a ingestão diária de energia $(\mathrm{kcal} / \mathrm{kg})$ e proteína $(\mathrm{g} / \mathrm{kg})$.

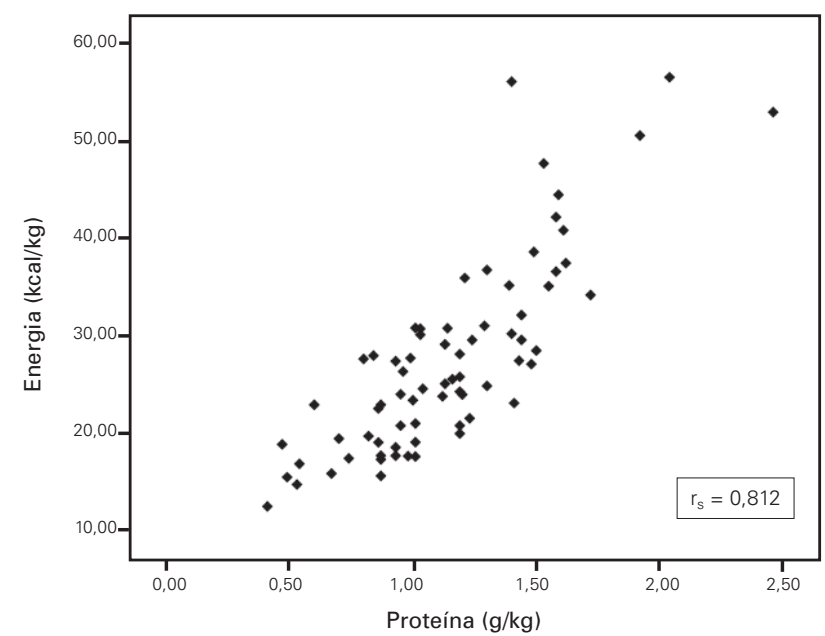


Observou-se correlação negativa baixa entre a ingestão energética e de proteína de AVB. Entre a ingestão de proteína (g e g/kg) houve uma correlação positiva baixa, e foi encontrada uma correlação positiva substancial na porcentagem do VCT. A correlação do fósforo diário e da proteína de AVB foi positiva baixa (Gráfico 3).

A ingestão de fósforo (Gráfico 4) apresentou correlação positiva forte com a ingestão proteica (g e g/ $\mathrm{kg}): 0,822(\mathrm{p}<0,01)$ e $0,753(\mathrm{p}<0,05)$, respectivamente. Ela também foi correlacionada com o VCT $\left(\mathrm{r}_{\mathrm{s}}=0,24, \mathrm{p}<0,01\right)$.

Com relação aos exames bioquímicos, a média do fósforo sérico foi de $5,9 \pm 1,9 \mathrm{mg} / \mathrm{dL}$ e a média do $\mathrm{Kt} / \mathrm{V}$ foi de $1,40 \pm 0,53$.

Gráfico 3. Coeficiente de correlação de Spearman entre a ingestão diária de proteína de alto valor biológico (\%) e fósforo (mg/dia).

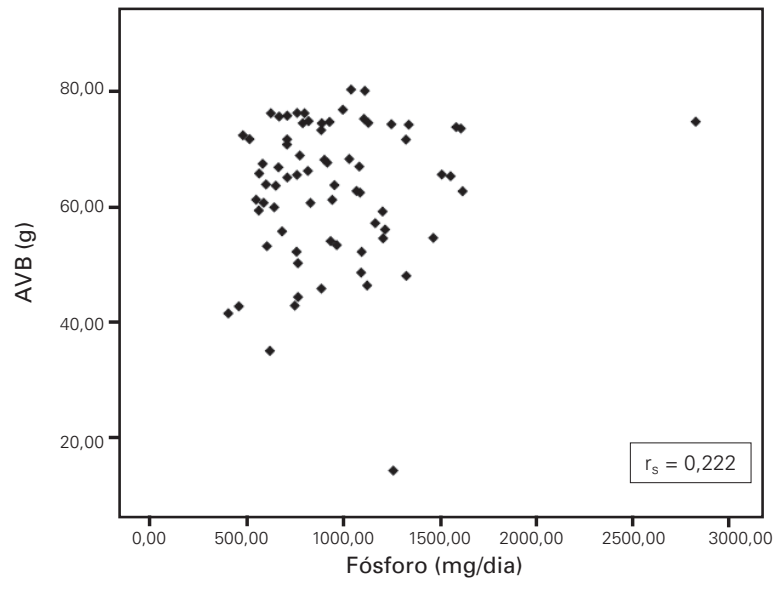

Gráfico 4. Coeficiente de correlação de Spearman entre a ingestão diária de proteína $(\mathrm{g} / \mathrm{kg})$ e fósforo $(\mathrm{mg} / \mathrm{dia})$.

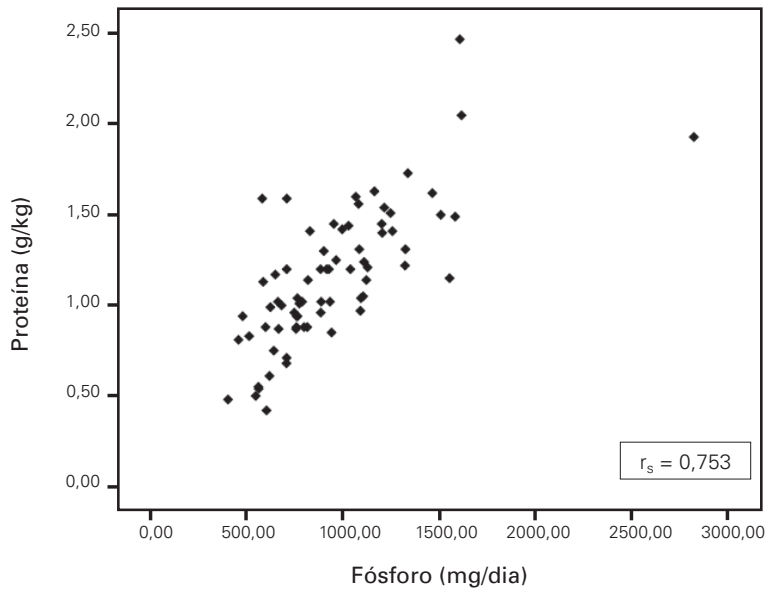

\section{DIscussÃo}

Os objetivos dietoterápicos no tratamento dialítico buscam auxiliar no controle e na prevenção das complicações da DRC, associadas às alterações metabólicas e à ingestão inadequada de macro e micronutrientes, visando à melhora do estado nutricional e físico do paciente.

Além dos fatores catabólicos inerentes à DRC e aos métodos dialíticos, a presença de DEP está comumente associada ao baixo consumo alimentar. ${ }^{17}$ A prevalência de DEP na população de pacientes em diálise é bastante elevada, variando de $23 \%$ a $76 \%$ nos pacientes em HD e de $18 \%$ a $50 \%$ nos pacientes de CAPD. ${ }^{18}$ Peters, Jorgetti e Martini relacionaram o tempo de HD e o IMC dos pacientes e observaram que os menores índices de IMC estão associados ao maior tempo de $\operatorname{HD}(10,0 \pm 2,2$ anos), concluindo que esse pode interferir energeticamente no estado nutricional do paciente renal. ${ }^{19}$ Entretanto, no presente estudo, esse dado não foi avaliado. A média do IMC foi de $25 \pm 5 \mathrm{~kg} / \mathrm{m}^{2}$, sendo observado apenas $3 \%$ dos pacientes com baixo peso $\left(\mathrm{IMC}<18,9 \mathrm{~kg} / \mathrm{m}^{2}\right)$.

A diálise inadequada pode causar variações no apetite do paciente, uma vez que está associada a manifestações clínicas relacionadas com a uremia. No presente estudo, a maioria dos pacientes apresentou apetite normal e a média do Kt/V estava dentro do que o K/DOQI preconiza como adequado. Em comparação com estudos realizados nos Estados Unidos $(\mathrm{Kt} / \mathrm{V}=1,26)$ e no Japão $(\mathrm{Kt} / \mathrm{V}=1,37)$, a média observada no presente estudo está acima, mostrando que a adequação de diálise está correta. ${ }^{20}$ No entanto, um dos principais problemas na utilização do Kt/V é a coleta inadequada da ureia pré- e pós-diálise, que pode ocasionar resultados pouco confiáveis. ${ }^{11}$ Além disso, Kuhlmann et al. ${ }^{21}$ demonstraram que pacientes de maior superfície corporal e peso têm dificuldades de atingir as metas de $\mathrm{Kt} / \mathrm{V}$ e que isso é comum em alguns países.

As perdas de nutrientes durante o procedimento hemodialítico podem ser um fator importante para a desnutrição, uma vez que são perdidos, primariamente, aminoácidos, peptídeos e vitaminas hidrossolúveis. A cada sessão de HD de baixo fluxo são perdidos em torno de 5 a 8 g de aminoácidos livres e de 4 a $5 \mathrm{~g}$ ligados. $^{22,23}$ Dessa forma, a recomendação proteica é de $1,2 \mathrm{~g} / \mathrm{kg} / \mathrm{dia}$ e tem o objetivo de manter o balanço nitrogenado positivo ou neutro, podendo ser maior dependendo do nível de estresse e das necessidades metabólicas aumentadas do paciente. 
O consumo de proteínas de pacientes em HD tem sido relatado como abaixo das recomendações vigentes. Cuppari et al. avaliaram o perfil alimentar de 10 centros e observaram um consumo médio de 1,03 \pm $1,43 \mathrm{~g} / \mathrm{kg} / \mathrm{dia}$. Entretanto, em cinco dos 10 centros de HD estudados, o consumo proteico foi inferior a 1,0 $\mathrm{g} / \mathrm{kg} / \mathrm{dia} .{ }^{24}$ No estudo realizado por Peters et al. foram avaliados 26 pacientes adultos com IRC, separados em dois grupos conforme os níveis de paratormônio (PTH), e foi observada média de consumo de proteína de $0,9 \pm 0,5 \mathrm{~g} / \mathrm{kg} / \mathrm{dia}$ em um grupo e $1,2 \pm 0,4$ no outro. No presente estudo, como nos descritos anteriormente, observou-se uma ingestão de $1,1 \pm 0,4 \mathrm{~g} /$ $\mathrm{kg} / \mathrm{dia}$, apontando que os pacientes apresentaram ingestão abaixo das recomendações, havendo risco de desnutrição. ${ }^{19}$

A qualidade da proteína também é relevante, sendo priorizados no mínimo $50 \%$ de proteínas AVB, com a finalidade de assegurar a ingestão de aminoácidos essenciais. ${ }^{1,12}$ Neste estudo, a quantidade de proteínas de AVB observada estava dentro do recomendado. É possível que, com as orientações repassadas pelo nutricionista nos serviços de HD, os pacientes façam escolhas inteligentes, ingerindo proteínas de AVB.

Com relação à ingestão energética, a recomendação é de $35 \mathrm{kcal} / \mathrm{kg}$ para a manutenção do peso e o balanço nitrogenado neutro em pacientes clinicamente estáveis em HD. ${ }^{12}$ Hylander et al. postularam que pacientes em estágio terminal da DRC, tratados com HD e DP, demonstram um padrão alterado na ingestão de alimentos, sendo, em média, de 22,8 $\pm 8,8 \mathrm{kcal} / \mathrm{kg} /$ dia. ${ }^{25}$ No presente estudo, a média da ingestão energética foi de $28 \pm 10 \mathrm{kcal} / \mathrm{kg} / \mathrm{dia}$, indicando que, apesar da ingestão acima do identificado em outros estudos, ela ainda está abaixo das recomendações. Esse fato pode estar associado a alguns fatores consequentes da HD, como anorexia, doenças intercorrentes, gastroparesia, anemia, inadequação dialítica, depressão e inflamação. Contudo, os registros alimentares possuem limitações e podem apresentar erros, uma vez que informam dados subjetivos que dependem da descrição correta da real ingestão alimentar. De qualquer forma, o aconselhamento dietético pelo profissional nutricionista é fundamental, visando a identificar erros alimentares e deficiências nutricionais. ${ }^{26}$

Estudos demonstram que existe uma ingestão deficiente de energia e proteínas em pacientes em HD. Além disso, sugerem que a ingestão calórica abaixo do recomendado é mais comum e grave do que a baixa ingestão proteica, o que foi encontrado no presente estudo. Estudos recentes demonstraram que o status nutricional é mantido mesmo quando a ingestão diária de proteínas está abaixo das recomendações do K/DOQI. ${ }^{26,27}$ Segundo Ichikawa, quando a ingestão calórica for de $35 \mathrm{kcal} / \mathrm{kg}$ ou mais, a ingestão proteica de $0,9 \mathrm{~g} / \mathrm{kg} / \mathrm{dia}$ é suficiente para manter o balanço nitrogenado neutro nos pacientes. ${ }^{28}$ Entretanto, é necessário ressaltar que a ingestão abaixo de $1,2 \mathrm{~g} / \mathrm{kg}$ não é preconizada pelo K/DOQI. Portanto, novos estudos devem ser realizados para evidenciar as melhores recomendações proteicas para os pacientes, pois, em muitos estudos, o valor preconizado não é atingido. Entretanto, devem ser avaliados os métodos de investigação dos estudos que podem conter falhas.

Com relação ao teor energético da dieta, uma ingestão inadequada resulta em alterações na composição corporal em decorrência da diminuição do tecido adiposo e da massa muscular. O baixo consumo também pode comprometer a utilização da proteína ingerida pelo aumento da gliconeogênese e promover aumento na formação de produtos residuais do metabolismo proteico que podem agravar os sintomas urêmicos, além de estimular o catabolismo dos tecidos corporais. ${ }^{29}$ No presente estudo, observou-se correlação positiva forte entre a ingestão energética $(\mathrm{kcal} / \mathrm{kg})$ e proteica $(\mathrm{g} / \mathrm{kg})$, evidenciando que, quanto maior a ingestão energética, maior será a ingestão proteica. ${ }^{25}$ Entretanto, é importante salientar que as recomendações do K/DOQI não foram atingidas pelos pacientes, o que pode estar associado a outros fatores não coletados neste estudo, como perfis socioeconômico e psicológico. $^{30}$

A recomendação dietética de fósforo é de 800 a $1.000 \mathrm{mg} /$ dia e deve ser individualizada, pois depende de fatores como fase da doença, uso de calcitriol, tipo de doença óssea e concentração sérica de fósforo. ${ }^{12} \mathrm{~A}$ HD não é considerada um método eficiente para a remoção do excesso do fósforo retido e, por isso, o cálculo desse elemento na dieta é fundamental, visando à homeostase do cálcio e do fósforo para manutenção da massa óssea. Entretanto, como a recomendação para pacientes em HD é uma dieta hiperproteica e os alimentos com alto teor de proteína são naturalmente ricos em fósforo, isso pode contribuir para elevação do fósforo ingerido, cujo cálculo adequado é um desafio. ${ }^{26,31}$

A média da ingestão diária observada no presente estudo foi de $958 \pm 374 \mathrm{mg} / \mathrm{dia}$, indicando uma ingestão adequada. Entretanto, essa ingestão pode ter ocorrido pela baixa quantidade de proteínas da dieta, fato que pode ter sido subestimado pelos pacientes. ${ }^{12,26}$ Além disso, também foi observada correlação positiva forte entre a ingestão de fósforo $(\mathrm{mg} / \mathrm{dia})$ 
e a ingestão energética $(\mathrm{kcal} / \mathrm{kg})$ e proteica $(\mathrm{g} / \mathrm{kg})$, de 0,824 e 0,753 respectivamente. Os alimentos de origem vegetal e animal (proteína de AVB) são fontes de fósforo. A biodisponibilidade é maior nos produtos de origem animal $(70 \%)$ que nos de origem vegetal. Com relação à proteína de AVB e ao fósforo (mg/dia), observou-se correlação significativa positiva baixa, demonstrando que o consumo de fósforo dietético não está apenas relacionado com alimentos de AVB. ${ }^{32,33}$ Portanto, é necessário ter cautela quanto a outras fontes alimentares na prescrição dietética do paciente em $\mathrm{HD}$, e não apenas com relação à proteína AVB.

Em relação ao fósforo sérico, os valores encontrados estão acima do preconizado, apesar da baixa ingestão de proteínas. Por outro lado, há variáveis importantes a considerar, como a qualidade da membrana do dialisador, o uso de aditivos alimentares com fosfato e a não total aderência dos pacientes aos quelantes de fosfato. Estudos demonstraram que menos de $25 \%$ dos pacientes em diálise aderem à dieta e aos medicamentos prescritos. ${ }^{34}$ Porém, a adesão aumenta se houver apoio de uma equipe profissional e se os pacientes apresentarem conhecimento sobre uma dieta adequada..$^{30}$ Além disso, mostrou-se que os pacientes que compreenderam as consequências da hiperfosfatemia mantêm o fósforo sérico mais baixo do que os demais. ${ }^{35}$

Outros estudos mostraram que o fósforo sérico acima de $6,2 \mathrm{mg} / \mathrm{dL}$ aumenta os níveis de PTH e que pode estar associado ao risco de mortalidade no paciente portador de DRC. ${ }^{36,37}$ Neste estudo, a maioria dos pacientes não utilizaram quelantes de fósforo $(79,2 \%)$, indicando que o uso do medicamento não alterou os achados bioquímicos. Segundo Saran et al., os níveis séricos de fósforo acima de $7,5 \mathrm{mg} / \mathrm{dL}$ são provavelmente um reflexo da não aderência à dieta e/ ou aos medicamentos, em comparação com os níveis séricos de $5,5 \mathrm{mg} / \mathrm{dL}$ e $6,5 \mathrm{mg} / \mathrm{dL}^{27}$

O tratamento nutricional, visando à oferta de dieta adequada em energia, proteína e fósforo, associado à diálise adequada e à terapêutica medicamentosa, é fundamental para auxiliar no controle e na prevenção de DEP, hiperfosfatemia e outras alterações metabólicas que levam aos sintomas urêmicos.

\section{Conclusão}

Há associação positiva entre a ingestão de energia, proteína e fósforo na alimentação de pacientes em HD. O controle desses nutrientes na dieta, associado à diálise adequada, é essencial para evitar as complicações relacionadas com a DRC, como DEP, anorexia, hiperfosfatemia, hiperparatireoidismo secundário e doença óssea metabólica. Pelo risco nutricional apresentado por esses pacientes - em grande parte por uma ingestão alimentar deficiente -, algumas estratégias podem ser utilizadas para melhorar a aderência à dieta, sendo essencial a apresentação das razões e dos efeitos das recomendações. Portanto, a vigilância e a intervenção nutricional, como a boa diálise e o uso da terapêutica medicamentosa nos pacientes portadores de DRC, são importantes nos serviços de diálise, visando a avaliar o perfil dietético dos pacientes para corrigir erros alimentares.

\section{REFERÊNCIAS}

1. Santos PR, Coelho MR, Gomes NP et al. Associação de indicadores nutricionais com qualidade de vida em pacientes portadores de doença renal crônica em hemodiálise. J Bras Nefrol 2006; 28:57-64.

2. Schor N, Ajzen H. Insuficiência Renal Crônica. In: Schor, N e Ajzen, H. Guias de Medicina Ambulatorial e Hospitalar UNIFESP - Escola Paulista de Medicina - Nefrologia, $2^{a}$ edição. São Paulo: Manole, 2005, pp.183-4.

3. Bilbrey $\mathrm{C}$, Cohen T. Identification and treatment of protein calorie malnutrition in chronic hemodialysis patients. Nephrol Dial Transplant 1989; 18:669-78.

4. Gotch FA, Sargent JA. Mechanistic analysis of the National Cooperative Dialysis Study. Kidney Int 1985; 28:526-34.

5. Alvestrand A. Protein metabolism and nutrition in hemodialysis patients control. Nefrology 1990; 78:102-18.

6. Bastos MG, Carmo WB, Abrita RR et al. Doença renal crônica: problemas e soluções. J Bras Nefrol 2004; 26: 202-15.

7. Beto JA, Bansal VK. Medical nutrition therapy in chronic kidney failure: Integrating clinical practice guidelines. J Am Dietetic Association 2004; 104:404-40.

8. Andress DL. Vitamin D treatment in chronic kidney disease. Seminars in Dialysis 2005; 18:315-21.

9. Bilbrey C, Cohen T. Identification and treatment of protein calorie malnutrition in chronic hemodialysis patients. Nephrol Dial Transplant 1989; 18:669-78.

10. Batista T, Vieira I, Azevedo L. Avaliação nutricional de pacientes em programa de hemodiálise crônica. J Bras Nefrol 2004; 26:113-20.

11. London GM, Guerin AP, Marchais SJ et al. Arterial media calcification in renal disease: impact on all-Cause and cardiovascular mortality. Nephrol Dial Transplant 2003; 18:1731-40.

12. National Kidney Foundation. NKF-DOQI - clinical practice guidelines for nutrition in chronic renal failure. Am J Kidney Dis 2000; 35(Suppl 2):17-103.

13. Burmeister MM, Antonello ICF, Pizzato AC. Ingestão e níveis séricos de potássio, fósforo e cálcio de pacientes com insuficiência renal crônica em tratamento hemodialítico [dissertação]. Porto Alegre: PUCRS, 2008.

14. Schor N, Ajzen H. Técnicas Dialíticas na Insuficiência Renal Crônica. In: Guias de Medicina Ambulatorial e Hospitalar UNIFESP - Escola Paulista de Medicina Nefrologia, 2 ed. São Paulo: Manole, 2005, pp. 227-9. 
15. National Kidney Foundation, Updates Clinical Practice Guidelines and Recommendations, 2006. Acessado em: http://www.kidney.org/professionals/kdoqi/pdf/hemodialysis_adequacy.pdf

16. BRASIL. Conselho de Saúde. Diretrizes E Normas Regulamentadoras de Pesquisa em Seres Humanos (Resolução 196/96). Diário Oficial da União, 16 de outubro de 1996.

17. Bergstrom J. Why are dialysis patients malnourished. Am J Kidney Dis 1995; 26:229-41.

18. Khaja RA. Nutrition in maintenance hemodialysis patients in nutritional management of renal disease. Edited: Kopple JD, Massry SG. Baltimore: Williams \& Wilkins, 1997.

19. Peters BSE, Vanda \& Martini J, Araújo L. Influência do hiperparatireoidismo secundário grave no estado nutricional de pacientes com insuficiência renal crônica. Rev Nutr 2006; 19:111-8.

20. Lameire N. Management of the hemodialysis patient: an European perspective. In: Ronco C, Levin NW. Advances in end-stage renal disease; 2002. Basel: Karger, 2002, pp. 93-100.

21. Kuhlmann MK, Konig J, Riegel W, Kohler H. Genderspecific differences in dialysis quality $(\mathrm{Kt} / \mathrm{V})$ : "big men" are at risk of inadequate haemodialysis treatment. Nephrol Dial Transplant 1999; 14:147-53.

22. Batista T, Vieira I, Azevedo L. Avaliação nutricional de pacientes em programa de hemodiálise crônica. J Bras Nefrol 2004; 26:113-20.

23. London GM, Guerin AP, Marchais SJ et al. Arterial media calcification in renal disease: impact on all-Cause and cardiovascular mortality. Nephrol Dial Transplant 2003; 18:1731-40.

24. Cuppari L, Draibe AS. Avaliação nutricional de pacientes renais crônicos em programa de hemodiálise: estudo multicêntrico. Rev Assoc Med Bras 1989; 35:9-14.

25. Hylander B, Barkeling B, Rossner S. Eating behavior in continuous peritoneal dialysis and hemodialysis patients. Am J Kidney Dis 1992; 20:592-7.

26. Cabral PC, Diniz AS, Arruda IKG. Avaliação nutricional de pacientes em hemodiálise. Rev Nutr 2005; $18: 29-40$
27. Saran R, Bragg-Gresham JL, Rayner HC et al. Nonadherence in hemodialysis: associations with mortality, hospitalization, and practice patterns in the DOPPS. Kidney Int 2003; 64:254-62.

28. Ichikawa Y, Hiramatsu F, Hamada $\mathrm{H}$ et al. Effect of protein and energy intakes in body composition in nondiabetic maintenance in hemodialysis patients. J Nutr Sci Vitaminol 2007; 53:410-8.

29. Fausto MA, Gomes JG, Iglesias ACRG et al. Avaliação Nutricional de pacientes com insuficiência renal crônica submetidos à hemodiálise. Alim Nutr São Paulo 1996; 7:15-23.

30. Hoover HH. Compliance in hemodialysis patients: a review of the literature. J Am Diet Assoc 1989; 89:957-9.

31. Rufino M, de Bonis E, Martin M et al. Is it possible to control hyperphosphataemia with diet, without inducing protein malnutrition? Nephrol Dial Transplant 1998; 13(Suppl 3):65-7.

32. Bour NJS, Soullier BA, Zemel MB. Phosphate transport into brush-border membrane vesicles isolated from rat small intestine. Biochem J V 1976; 160:467-74.

33. Shapiro A, Heaneyb RP. Co-dependence of calcium and phosphorus for growth and bone development under conditions of varying deficiency. Bone 2003; 32:532-40.

34. Marchais SJ, Metivier F, Guerin AP, London GM. Association of hyperphosphataemia with haemodynamic disturbances in end-stage renal disease. Nephrol Dial Transplant 1999; 14:2178-83.

35. Thomas LK, Sargen RG, Michels PC, Richter DL, Valois RF, Moore CG. Indentification of the factors associated with compliance to therapeutic diets in alder adults with end stage renal disease. J Ren Nutr 2001; 11:80-9.

36. Stamatiks MK, Pecora PG, Gunel E. Factors influencing adherence in chronic dialysis patients with hyperphosphatemia. J Ren Nutr 1997; 7:144-8.

37. Block GA, Hulbert-Shearon TE, Levin NW et al. Association of serum phosphorus and calcium $\mathrm{x}$ phosphate product with mortality risk in chronic hemodialysis patients: a national study. Am J Kidney Dis 1998; 31:607-17. 